\title{
O PROBLEMA DOS FUNDAMENTOS DA MATEMÁTICA E AS RAÍZES DO ANTI-EXCEPCIONALISMO LÓGICO
}

\author{
Sanderson Molick ${ }^{1}$
}

\begin{abstract}
RESUMO
A chamada crise dos fundamentos da matemática, perpetrada pela descoberta dos paradoxos da teoria ingênua de conjuntos, bem como pela descoberta dos teoremas de Gödel, desencadeou uma série de reações à forma como entendemos o conhecimento matemático. Dentre as visões usuais, as mais conhecidas tentam erguer uma base para o conhecimento matemático a partir de elementos a priori, como a noção de prova, ou de "intuição primordial", como é o caso de alguns intuicionistas. O presente artigo tem como objetivo apresentar uma visão antagônica às posições apriorísticas como forma de identificar as raízes históricas do chamado anti-excepcionalismo lógico. Para tanto, o texto apresenta as ideias de Lázslo Kalmár e de seu empirismo matemático com o objetivo de contrastar formas de anti-excepcionalismo pré- e pós-Quine. O problema dos fundamentos da matemática é utilizado como critério de demarcação entre estas posições.
\end{abstract}

Palavras-chave: Anti-Excepcionalismo Lógico. Fundamentos da Matemática. Lázslo Kalmár. Empirismo Matemático.

\begin{abstract}
The so-called foundational crisis started by the discovery of the set-theoretic paradoxes in naive set theory, as well as by the discovery of Gödel's incompleteness theorems, was responsible for a series of reactions over the way we understand mathematical knowledge. Among the common views, the most popular ones try to construct mathematical knowledge on a priori elements, such as the notion of proof, or even ideas of "primordial intuitions", as defended by some intuitionists. The purpose of the present paper is to introduce opposite views to the traditional aprioristic ones as a way to trace the historical roots of the so-called anti-exceptionalism about logic. In order to do so, the paper presents Lázslo Kalmár's ideas over his mathematical empiricism as a way to contrast two types of anti-exceptionalism, namely pre- and post-

\footnotetext{
${ }^{1}$ Doutorando em Filosofia pela Universidade Federal do Rio Grande do Norte/UFRN e a Ruhr-Universität Bochum/RUB.

E-mail:smolicks@gmail.com. ORCID: 0000-0003-2918-1055.
} 
Quine. The problem of mathematical foundations is explored as a demarcation criterion between these positions.

Key-words: Logical Anti-Exceptionalism. Foundations of Mathematics. Lázslo Kalmár. Mathematical Empiricism.

\section{Introdução}

O anti-excepcionalismo lógico afirma que a lógica não é uma exceção entre as ciências empíricas. Os métodos de teste e decisão das teorias e proposições lógicas são semelhantes aos métodos de teste e decisão das teorias científicas. Com isso, de acordo com o anti-excepcionalismo, a evidência empírica também desempenha um papel importante na justificação dos princípios lógi$\cos$.

O presente artigo examina as raízes históricas do anti-excepcionalismo lógico. Embora o termo anti-excepcionalismo tenha sido cunhado apenas recentemente por Timothy Williamson [Williamson, 2013], a ideia de que a lógica não difere das ciências empíricas remete também aos empiristas matemáticos da primeira metade do século XX. Dentre eles, encontramos autores como John Stuart Mill, Bertrand Russell, Fraenkel, Georg Kreisel e Lázslo Kalmár.

$\mathrm{O}$ artigo divide o anti-excepcionalismo historicamente em duas fases. Como critério de demarcação entre os anti-excepcionalistas da primeira fase e os anti-excepcionalistas atuais, o artigo utiliza o problema dos fundamentos e da justificação do conhecimento matemático como forma de sublinhar as diferenças entre as duas formas de anti-excepcionalismo. Portanto, o uso do termo anti-excepcionalismo estará ligado ao uso e aceitação de evidência a posteriori como forma de justificação do conhecimento, seja do nosso conhecimento dos princípios da lógica, ou do conhecimento das verdades matemáticas. ${ }^{2}$ No percurso de desenvolvimento do artigo, a expressão problema dos fundamentos se referirá ao problema de encontrar fundamentos apriorísticos para o conhecimento matemático. Programa iniciado a partir dos esforço logicista de Frege e

\footnotetext{
${ }^{2}$ Note, contudo, que o artigo não pretendo colapsar o empirismo matemático ao anti-excepcionalismo lógico, mas apenas contrastar ambas posições a partir de um ponto em comum, a saber, da aceitação da evidência empírica como forma de justificação de suas verdades.
} 
sucedido por diversos programas de pesquisa, como o formalismo de Hilbert e o intuicionismo de Brouwer.

$\mathrm{Na}$ primeira forma de anti-excepcionalismo, aqui denominada de antiexcepcionalismo pré-quineano, o caráter não-apriorístico da lógica implica repensar o problema dos fundamentos da matemática a partir da admissão de que certas áreas da matemática possuem validação empírica. Na segunda fase, aqui denominada de anti-excepcionalismo pós-quineano, o caráter não-apriorístico da lógica é utilizado como via de solução para o problema da rivalidade entre lógicas, de modo a fazer uso de modelos abdutivos como processo de decisão entre teorias lógicas rivais.

Uma forma de anti-excepcionalismo pré-quineano é ilustrada a partir de Lázslo Kalmár, autor central na defesa e formulação do empirismo matemático como via de solução ao problema dos fundamentos. Para tanto, o artigo apresenta uma descrição da filosofia da matemática de Kalmár e o situa em comparação às formas contemporâneas de anti-excepcionalismo lógico.

$\mathrm{O}$ artigo possui a seguinte estrutura: a seção 2 introduz o problema dos fundamentos e discute os problemas filosóficos que nascem desta; na seção 3 é introduzido o anti-excepcionalismo lógico. As diferentes formas de anti-excepcionalismo são classificadas em anti-excepcionalismos pré- e pósquineano. A distinção entre ambas formas de anti-excepcionalismo é introduzida a partir do modo como ambos vêem o problema dos fundamentos. Por fim, na seção 4, o anti-excepcionalismo de Kalmár é apresentado como exemplo paradigmático de anti-excepcionalismo pré-quineano. Os aspectos centrais de sua filosofia da matemática são apresentados e discutidos.

\section{O problema dos fundamentos}

A lógica contemporânea se desenvolve do esforço logicista de fundamentar o conhecimento da aritmética nas leis e regras da lógica. É a partir dos trabalhos de Frege, em especial, em Os fundamentos da aritmética, que encontramos uma formulação dos axiomas e regras da, hoje chamada, lógica clássica. Se encontramos em Kant a defesa de que o conhecimento matemático se origina nas intuições puras do tempo e do espaço, há em Frege a tentati- 
va de se demonstrar que o conhecimento da aritmética pode ser fundamentado na lógica. O programa logicista, conforme já conhecido, culmina na descoberta do paradoxo de Russell e desencadeia a chamada crise dos fundamentos.

Duas questões se tornam centrais para a filosofia da lógica e da matemática a partir da crise dos fundamentos, a saber: 1) qual a fonte do conhecimento matemático?, e 2) como podemos justificar o conhecimento matemático ou, em particular, como podemos justificar nosso conhecimento da aritmética? Uma das vias de solução buscou responder a segunda questão a partir de métodos apriorísticos, isto é, utilizando os métodos da lógica para demonstrar como nosso conhecimento da matemática pode ser reduzido a princípios lógicos fundamentais. Para eles, mostrar que o conhecimento matemático pode ser fundado em princípios lógicos significava demonstrar o caráter infalível da matemática. Assim, a matemática fixaria seu lugar como uma disciplina dotada de um grau de certeza maior que as ciências empíricas, isto é, a matemática seria uma exceção dentre as outras ciências.

Uma outra via de solução, aqui chamada de anti-excepcionalista, enxergava o conhecimento matemático enquanto revisável e passível de refutação empírica. Para muitos destes autores, os resultados matemáticos jamais poderiam ser assentados sobre princípios sólidos e indubitáveis. Em [Lakatós, 1976], encontramos um registro de alguns dos autores que partilhavam esta visão, como Fraenkel, Church, Weyl, dentre outros. Tal visão sobre o conhecimento da matemática era denominada de empirismo matemático. No que segue, apresento o empirismo matemático tal como encontrado na visão de um autor central deste período, Lázslo Kalmár.

\section{A solução anti-excepcionalista}

Em sua acepção moderna, o anti-excepcionalismo lógico é caracterizado por [Hjortland, 2016] a partir de duas teses centrais: 1) a lógica não é a priori; e 2) as teorias lógicas podem ser escolhidas a partir do mesmo processo de decisão utilizado para escolher entre teorias científicas rivais, isto é, a partir da comparação das virtudes epistêmicas de cada teoria. Dentre estas 
virtudes, encontramos a consistência, a adequação aos dados, o poder explicativo, a fecundidade etc.

No presente artigo dividirei os anti-excepcionalistas a partir de dois momentos centrais: os anti-excepcionalistas da era pré-quineana e os antiexcepcionalistas da era pós-quineana. Dentre os do segundo grupo, destaco [Williamson, 2013], [Priest, 2016] e [Hjortland, 2016]. Já entre os primeiros, é possível classificar autores que vão desde John Stuart Mill à Georg Kreisel, Lazslo Kalmár e Imre Lakatos. Contudo, por razões de espaço (e do fato de que ainda é difícil o acesso à obra de alguns destes outros autores), focarei apenas em Kalmár.

Influenciados pelos trabalhos de Quine e pelas questões da filosofia da lógica contemporânea, tais como a rivalidade entre lógicas, o pluralismo lógico e a validade dos princípios da lógica clássica, os trabalhos dos anti-excepcionalistas da era pós-quineana possuem maior foco na tese (2) acima e em como os métodos de escolha entre teorias científicas rivais podem ser aplicados na lógica. A escolha de Quine como ponto de inflexão está no fato de que tal autor se enquadra como um dos primeiros a utilizar critérios abdutivos como forma de defender a superioridade da lógica clássica em relação à algumas lógicas não-clássicas.

Em contrapartida, os anti-excepcionalistas da era pré-quineana se caracterizam por uma maior preocupação na tese (1), isto é, em como caracterizar adequadamente o caráter não-apriorístico da lógica e no que isto representaria para a forma como justificamos o conhecimento matemático. Deste modo, enquanto os anti-excepcionalistas da era pós-quineana acreditam que encontrar um fundamento adequado para a matemática significa encontrar a melhor teoria lógica dentre as concorrentes, os anti-excepcionalistas da era pré-quineana acreditam que o caráter não-apriorístico da lógica, por si só, demonstra que a matemática não precisa de uma teoria lógica para a justificação de seus princípios.

Os anti-excepcionalistas da era pós-quineana encontram no caráter não-apriorístico da lógica uma via de saída para o problema da rivalidade entre lógicas. Estes autores ainda reconhecem a necessidade de encontrar funda- 
mentos adequados para as teorias matemáticas, para as teorias da verdade, as teorias do significado, etc. Assim, se encontramos uma variedade de sistemas lógicos disponíveis e nos perguntamos qual destes deveria fundamentar certas áreas do conhecimento, os anti-excepcionalistas da era pós-quine defendem que podemos escolher cada sistema a partir do uso de modelos abdutivos de escolha.

É importante ressaltar, contudo, que a noção de evidência utilizada pelos autores da era pré-quineana não é a mesma da era pós-quineana. Embora seja verdade que ambos os grupos aceitam o papel da evidência a posteriori na lógica e na matemática, é somente nos autores da era pós-quineana que encontramos a aceitação da evidência psicológica e de outras formas de evidência baseadas em dados não-observacionais. Uma das razões para tal diferença entre as concepções de evidência reside no fato de que as discussões contemporâneas acerca da falibilidade de certos princípios da lógica clássica parecem aceitar uma ampla gama de dados como forma de justificar determinadas lógicas não-clássicas. Desse modo, é comum encontrarmos dados diferentes como situações epistêmicas, construções matemáticas, discussões metafísicas etc, como forma de legitimar a falibilidade de um princípio clássico em favor de sua revisão ${ }^{3}$.

Conforme mecionado, encontramos em Kalmár, e em certa medida também em Lakatos, a crença de que o caráter não-apriorístico da lógica revela apenas que o problema de encontrar fundamentos seguros para a matemática não se coloca da maneira usual, pois os axiomas e os princípios lógicos partem da experiência empírica e são validados por esta. É verdade que, por razões históricas óbvias, estes autores não lidam com o problema da rivalidade entre lógicas. Contudo, é importante ressaltar que, diferentemente dos autores da era pós-quineana, a crença no caráter empírico da lógica conduz estes autores à uma visão distinta sobre o problema dos fundamentos. Na seção seguinte, será exposta a visão de Kalmár acerca do conhecimento matemático.

${ }^{3}$ Para mais sobre este ponto, ver [Molick, 2020]. 


\section{O anti-excepcionalismo de Kalmár}

Conforme discutido, as raízes do anti-excepcionalismo lógico se encontram no empirismo matemático defendido por muitos filósofos e matemáticos da primeira metade do século XX. De acordo com o empirismo matemático, as verdades matemáticas são justificadas através da evidência empírica, assim como nas outras ciências. Uma figura que desempenha papel central na defesa e formulação de muitas das teses do empirismo matemático é o lógico húngaro Lazslo Kalmár (1905-1976).

Em [Kalmar, 1967], o autor argumenta que a invenção da abstração, da inferência dedutiva, e do método axiomático, foi responsável por grande parte do desenvolvimento da matemática. Contudo, tais métodos levaram os matemáticos a entender a matemática como uma disciplina "puramente dedutiva" em oposição às disciplinas que fazem uso de testes empíricos. De acordo com Kalmár, o método da abstração possui as seguintes funções centrais: 1) descobrir regularidades gerais entre os fatos empíricos, e 2) permitir a construção de conceitos idealizados, tais como os de ponto ou reta, para os quais existem apenas aproximações no mundo real, mas cuja simplicidade lógica os torna mais adequados para o uso e manipulação na inferência dedutiva.

Para Kalmár, a abstração é o primeiro passo em direção aos métodos de produção dos teoremas e resultados matemáticos. É a partir da abstração que conseguimos a via de acesso à axiomatização, conceitualização e até mesmo à construção de modelos. O percurso do conhecimento matemático é descrito por Kalmár em [Kalmár, 1942]. Conforme descreve o autor, o conteúdo de nossas intuições permite o matemático alcançar passos de abstração cada vez maiores. Assim, o conhecimento matemático parte da realidade empírica e produz intuições que serão refinadas a partir da conceitualização e axiomatização. É somente a partir daí que o conteúdo dos axiomas será abstraído de forma a produzir teorias matemáticas distantes da intuição. Tais teorias serão reguladas por princípios e regras básicas e é a partir delas que o matemático construirá diferentes modelos de teorias formais. Dentro deste percurso, a busca por rigor se distancia e sacrifica cada vez mais o papel das intuições que antes originaram a teoria. É este o ponto central da crítica de Kalmár - o fato 
de que, ainda que as teorias matemáticas possam se reduzir a jogos formais, elas se baseiam em intuições que estão calcadas no mundo empírico. Deste modo, entender a matemática como uma ciência puramente dedutiva levou os matemáticos a esquecer que os axiomas e regras foram abstraídos de regularidades empíricas, as quais já possuem validade porque assim foram confirmadas em nossas práticas inferenciais.

Em [Kalmár, 1962], Kalmár argumenta que vários fatores contribuíram para sustentar a visão da matemática enquanto ciência pura dedutiva, a saber: 1) a consolidação de uma teoria do a priori, segundo a qual não é necessário o teste de axiomas e regras de inferência intuitivamente evidentes;2) a visão popular de que a matemática é uma disciplina "infalível” (o que neste contexto significa não-revisável); 3) a ideia de que a matematica serve como modelo de ciência a ser buscado pelas outras áreas do conhecimento em razão de sua precisão; 4) o próprio sucesso da matemática e a extensão da aplicação do método de abstração, os quais levaram a descoberta de resultados e teoremas matemáticos cada vez mais abstratos.

Deste modo, como é possível confirmar, encontramos em Kalmár a ideia de que o método de justificação do conhecimento matemático não se reduz aos métodos a priori. Embora a matemática faça uso de procedimentos a priori na demonstração de seus resultados, o recurso primário do conhecimento matemático é a experiência empírica, a qual será abstraída para a descoberta de regularidades e generalizações. Conforme ressalta o autor: "(...) Por que não confessamos que a matemática, assim como as outras ciências, baseia-se, e deve ser testada, na pratica [experiência]?”4

Neste ponto, embora Kalmár aponte para o uso da evidência a posteriori no papel de justificação do conhecimento matemático, não é claro que tipo de evidência é aceita por Kalmár ou como ocorreria o processo de revisão das verdades matemáticas a partir de evidências empíricas 5 . Mais ainda, embora o autor forneça uma breve descrição do processo de evolução da empiria à abstração [Kalmár, 2011], assim fornecendo informações sobre sua epistemolo-

${ }^{4}$ [Kalmár, 1962, p. 7], tradução minha.

${ }^{5} \mathrm{O}$ autor reconhece, contudo, a necessidade de abordar tais questões e as menciona como problemas em aberto para um programa de pesquisa que busque desenvolver fundamentos empíricos para os fundamentos da matemática. 
gia da lógica, o mesmo não parece fornecer uma descrição dos mecanismos heurísticos do processo de revisão de teorias matemáticas ${ }^{6}$.

É com base em sua visão acerca da justificação do conhecimento matemático que Kalmár formulará sua crítica à obsessão formalista de encontrar fundamentos puramente dedutivos para a matemática. Para o autor, a consistência de muitas de nossas teorias formais é apenas "um fato empírico" garantido pelo fato de que se fundamentam em axiomas abstraídos do caráter empírico de nossas práticas inferenciais. Com isso, a busca por fundamentos puramente dedutivos acaba por esquecer o lugar de onde tais axiomas e regras de inferência foram abstraídos, isto é, dos fenômenos empíricos. Assim, a busca pelos fundamentos da matemática só faz sentido na medida em que se reconheça que muitos princípios matemáticos são validados empiricamente $\mathrm{e}$ podem ser revisados à luz de nova evidência. Para Kalmár, mesmo proposições como a tese de Church deve ser investigada como um problema empírico, o qual é passível de teste e revisão (ver [Kalmár, 1959]).

\subsection{Os fundamentos da matemática - para onde?}

A crítica de Kalmár em [Kalmár, 1962] resume-se em seu slogan principal: fundamentos da matemática - para onde a partir de agora? $?^{7}$ Para ele, o programa de pesquisa em torno dos fundamentos da matemática parte de premissas errôneas. Apesar da existência de diversos programas de pesquisa envolvidos na fundamentação da matemática, como por exemplo o intuicionismo, a teoria de tipos de Whitehead e Russell, e a teoria da prova hilbertiana, ainda se faz necessário desenvolver novas linhas de pesquisa a partir da admissão de que a matemática não é uma ciência pura dedutiva.

A saída apontada pelo autor é reconhecer a matemática enquanto ciência com fundamentos empíricos. Uma das consequência de tal admissão faria com que fosse possível admitir o raciocínio indutivo dentro da prática matemática. Para Kalmár, o raciocínio indutivo e o raciocínio dedutivo possuem alguns problemas intrínsecos. Por exemplo, ainda que o raciocínio induti-

\footnotetext{
${ }^{6}$ É possível encontrar a descrição de tais mecanismos heurísticos, bem como do tipo de evi dência aceita, em [Lakatos, 1976].

${ }^{7}$ No original "Foundations of mathematics - whither now?"
} 
vo sofra dos problemas usuais ligados ao fato de que não podemos garantir a verdade em todos os casos de uma afirmação universal, as afirmações existenciais demonstradas por dedução também sofrem dos problemas comuns apontados pelo intuicionistas.

Uma vez admitidos os fundamentos empíricos da matemática, ainda se seguiriam novos problemas ligados aos mecanismos heurísticos de confirmação e refutação dos teoremas matemáticos. Dentre eles, estaria o problema de definir quais são os "observáveis” aos quais as proposições matemáticas estariam sujeitas à confirmação ou refutação, bem como o problema de delimitar quais proposições matemáticas estariam sujeitas à testes empíricos e quais não estariam ${ }^{8}$. É a partir de tais questões que Kalmár avança parte dos problemas que farão parte da agenda atual dos anti-excepcionalistas lógicos. Aceitar o papel da evidência a posteriori dentro do processos de justificação das teorias lógicas e matemáticas leva aos problemas centrais enfrentados pelos antiexcepcionalistas da era pós-quineana.

\section{Considerações finais}

No presente artigo examinei o anti-excepcionalismo lógico a partir do problema dos fundamentos da matemática. Com isto, foi defendida uma classificação das formas de anti-excepcionalismo pré- e pós-quineana como meio de ressaltar as divergências entre os anti-excepcionalistas da segunda metade do século XX e os anti-excepcionalistas da filosofia da lógica atual. $\mathrm{O}$ artigo focou unicamente no pensamento de Lázslo Kalmár. Contudo, as comparações feitas aqui poderiam ser exploradas entre diferentes autores do empirismo lógico. Assim, permanece como trabalho futuro o desenvolvimento de uma investigação histórica de outros autores que compuseram os anti-excepcionalistas da fase pré-quineana. Também se faz necessário uma comparação do trabalho destes autores em torno de problemas como o de definir a noção de evidência empírica para a lógica e a matemática, a heurística de confirmação e refutação de tais teorias à luz de evidências a posteriori, bem como o

${ }^{8} \mathrm{O}$ problema de identificar os observáveis de confirmação e/ou refutação das proposições matemáticas será posteriormente tratado por Lakatos em A renaissance of empiricism in the recent philosophy of mathematics (The British Journal for the Philosophy of Science, 1976). 
papel de tais elementos em debates como o pluralismo lógico e o processo de escolha de teorias lógicas.

\section{Referências}

[Hjortland, 2016] Hjortland, O. T. (2016). Anti-exceptionalism about logic. Philosophical Studies, pages 1-28.

[Kalmár, 1959] Kalmár, L. (1959). An argument against the plausibility of Church's Thesis. In: Constructivity in Mathematics, North Holland-Amsterdam.

[Kalmar, 1967] Kalmar, L. (1967). Foundations of mathematics-whither now? In Studies in Logic and the Foundations of Mathematics, volume 47, pages 187-207. Elsevier.

[Kalmár, 2011] Kalmár, L. (2011). The development of mathematical rigor from intuition to axiomatic method. In Der Wiener Kreis in Ungarn/The Vienna Circle in Hungary, pages 269-288. Springer.

[Lakatos, 1976] Lakatos, I. (1976). A renaissance of empiricism in the recent philosophy of mathematics. The British Journal for the Philosophy of Science, 27(3):201-223.

[Molick, 2020] Molick, S. (2020). Logical theory revision through data underdetermination: an anti-exceptionalist exercise. A publicar em Principia: an international journal of epistemology.

[Priest, 2016] Priest, G. (2016). Logical disputes and the a priori. Princípios: Revista de Filosofia, 23(40):29-57.

[Szabó, 2018] Szabó, M. (2018). Kalmár's argument against the plausibility of Church's thesis. History and Philosophy of Logic, 39(2):140-157.

[Williamson, 2013] Williamson, T. (2013). Modal logic as metaphysics. Oxford University Press. 